\title{
`Supporting Information
}

\section{Strain and Biaxial Modulus of CdSe Cores in Nanocrystal Films}

For a system with no shear stresses, strain is related to stress as

$$
\begin{aligned}
& \varepsilon_{x x}=\frac{\sigma_{x x}-v\left(\sigma_{y y}+\sigma_{z z}\right)}{E} \\
& \varepsilon_{y y}=\frac{\sigma_{y y}-v\left(\sigma_{x x}+\sigma_{z z}\right)}{E} \\
& \mathcal{E}_{z z}=\frac{\sigma_{z z}-v\left(\sigma_{x x}+\sigma_{y y}\right)}{E}
\end{aligned}
$$

The in-plane tensile strain for the unrelaxed film is $\varepsilon_{x x}=\varepsilon_{y y}=\varepsilon$ and the isotropic in-plane stress is $\sigma_{x x}=\sigma_{y y}=\sigma$. With the normal stress $\sigma_{z z}=0$, it follows that $\varepsilon_{z z}=-\left[\frac{2 v}{1-v}\right] \mathcal{E}$, where $v$ is Poisson's ratio and $E$ is Young's modulus.

For $\{100\}$ planes the Raman shift is ${ }^{1}$

$$
\omega=\omega_{0}+\frac{p \varepsilon_{z z}+q\left(\varepsilon_{x x}+\varepsilon_{y y}\right)}{2 \omega_{0}}
$$

Therefore, $\omega=\omega_{0}+\left[\frac{q-\frac{p v}{1-v}}{\omega_{0}}\right] \mathcal{E}$ in the fully strained region, $\omega=\omega_{0}+\left[\frac{q-\frac{p v}{1-v}}{2 \omega_{0}}\right] \varepsilon$ in the partially relaxed delaminated regions near a channel crack, and $\omega_{0}$ for the completely delaminated region.

$$
\mathrm{We} \mathrm{use}^{2} p=-\omega_{\mathrm{LO}}{ }^{2}=-0.454 \times 10^{5} \mathrm{~cm}^{-2}, q=-1.8 \omega_{\mathrm{LO}}{ }^{2}=-0.817 \times 10^{5} \mathrm{~cm}^{-2} \text {, and } \omega_{0}=
$$
$213 \mathrm{~cm}^{-1}$. Consequently, Equation 4 can be written as

$$
\omega-\omega_{0}=\left[\frac{\omega_{0} v}{1-v}-1.8 \omega_{0}\right] \varepsilon
$$


Using the angle averaged value of $v=0.37$ for CdSe, as reported previously, ${ }^{3}$

$\omega-\omega_{0}=-258 \varepsilon\left(\right.$ in $\left.\mathrm{cm}^{-1}\right)$, and for $\varepsilon=+1 \%, \omega-\omega_{0}=-2.58 \mathrm{~cm}^{-1}$ in the completely strained region. For our film we measure a $6.4 \mathrm{~cm}^{-1}$ Raman shift $\left(\delta_{2}=6.4 \pm 1.5 \mathrm{~cm}^{-1}\right)$, which corresponds to a total core strain of $\sim 2.5 \%$. The strain in the entire film as derived from optical microscopy is $11.7 \%$.

The biaxial modulus for the CdSe core: $E_{\mathrm{CdSe}} /\left(1-v_{\mathrm{CdSe}}\right)=41.5 /(1-0.37)=65.9$ $\mathrm{GPa}$, using values of $41.5 \mathrm{GPa}$ and 0.37 for $E$ and $v$, respectively, for hexagonal CdSe. ${ }^{3}$ The in-plane stress is $\sigma=\varepsilon E /(1-v)=1.6 \mathrm{GPa}$ for $2.5 \%$ strain. Thus the biaxial modulus for the film, $E_{\text {film }} /\left(1-v_{\text {film }}\right)=0.025 / 0.117 E_{\mathrm{CdSe}} /\left(1-v_{\mathrm{CdSe}}\right)=0.21 E_{\mathrm{CdSe}} /\left(1-v_{\mathrm{CdSe}}\right) \sim$ 13.8 GPa. For $v_{\text {film }}=0.1-0.5$, we obtain $E_{\text {film }}=6.8-12.4 \mathrm{GPa}$

From the micromechanics models discussed in the next section it is reasonable to take $E_{\mathrm{film}} /\left(1-v_{\mathrm{film}}\right)=2.75 E_{\mathrm{TOPO}} /\left(1-v_{\mathrm{TOPO}}\right)$. From this, $E_{\mathrm{TOPO}} /\left(1-v_{\mathrm{TOPO}}\right)=$ $(0.21 / 2.75) E_{\mathrm{CdSe}} /\left(1-v_{\mathrm{CdSe}}\right)=0.077 E_{\mathrm{CdSe}} /\left(1-v_{\mathrm{CdSe}}\right)=0.077 \times 65.9 \mathrm{GPa}=5.1 \mathrm{GPa}$. For $v_{\mathrm{TOPO}}=0.1-0.5$, we see that $E_{\mathrm{TOPO}}=2.45-4.41 \mathrm{GPa}$.

\section{Micromechanics Models}

Assuming a close-packed arrangement of spheres, the volume density of the CdSe cores in the film is assumed to be $\rho=0.43$. We use this and assume $E_{\mathrm{CdSe}}>E_{\mathrm{TOPO}}$ in each of the below micromechanics models, and find this assumption is consistent with the results.

\subsection{Halpin-Tsai Model}

This model predicts ${ }^{4}$ 


$$
\frac{E_{f i l m}}{E_{\text {TOРO }}}=\frac{1+\xi \eta \rho}{1-\eta \rho}
$$

with $\xi=2+40 \rho^{10}$ for spherical particles, where $\rho$ is the fraction of the particulate material in the matrix and

$$
\eta=\frac{\frac{E_{f i l m}}{E_{T O P O}}-1}{\frac{E_{f i l m}}{E_{\text {TOPO }}}+\xi}
$$

$E$ and $K$ scale in the same way, $E_{\text {film }} / E_{\mathrm{TOPO}}=K_{\text {film }} / K_{\mathrm{TOPO}}=3.26$. Therefore, $E_{\text {film }} /\left(1-v_{\text {film }}\right)$ $=3.26 E_{\mathrm{TOPO}} /\left(1-v_{\mathrm{TOPO}}\right)$.

\subsection{Cohen-Ishai Model}

This model predicts ${ }^{5}$

$$
E_{\text {film }}=E_{\text {TOPO }}\left(1+\frac{\rho}{\frac{M}{M-1}-\rho^{1 / 3}}\right)
$$

where $M=E_{\mathrm{CdSe}} / E_{\mathrm{TOPO}}$, to give $E_{\mathrm{film}} / E_{\mathrm{TOPO}}=2.75$. Using the assumptions of cubic filler in a cubic matrix implicit in this model with $v=0.33$, gives $E_{\text {film }} /\left(1-v_{\text {film }}\right)=2.75 E_{\mathrm{TOPO}} /(1-$ $\left.v_{\mathrm{TOPO}}\right)$.

\subsection{Mori-Tanaka Model}

This model predicts ${ }^{6}$

$$
\frac{E_{f i l m}}{E_{\text {TOPO }}}=1+\frac{\rho}{(1-\rho)}\left[1+\frac{\left(9 K_{\text {TOPO }}+8 E_{\text {TOPO }}\right)}{6\left(K_{\text {TOPO }}+2 E_{\text {TOPO }}\right)}\right]
$$




$$
\frac{K_{\text {film }}}{K_{\text {TOPO }}}=1+\frac{\rho}{(1-\rho)}\left[1+\frac{4 E_{\text {TOPO }}}{3 K_{\text {TOPO }}}\right]
$$

We examine two reasonable scenarios:

i) For $E_{T O P O}=K_{T O P O}$, we have $E_{\text {film }} / E_{\mathrm{TOPO}}=2.46$ and $K_{\mathrm{film}} / K_{\mathrm{TOPO}}=2.76$, and therefore $E_{\text {film }} /\left(1-v_{\text {film }}\right)=2.33 E_{\mathrm{TOPO}} /\left(1-v_{\mathrm{TOPO}}\right)$.

ii) For $E_{m}=0.5 K_{m}$, we have $E_{\mathrm{film}} / E_{\mathrm{TOPO}}=2.57$ and $K_{\mathrm{film}} / K_{\mathrm{TOPO}}=2.25$, and therefore $E_{\text {film }} /\left(1-v_{\text {film }}\right)=2.65 E_{\mathrm{TOPO}} /\left(1-v_{\mathrm{TOPO}}\right)$.

\subsection{Christensen-Lo Model}

This model predicts ${ }^{6}$

$$
K_{\text {film }}=K_{\text {TOPO }}+\frac{\rho\left(K_{\text {CdSe }}-K_{\text {TOPO }}\right)}{1+(1-\rho) \frac{\left(K_{\text {CASe }}-K_{\text {TOPO }}\right)}{\left(K_{\text {TOPO }}+\frac{4}{3} E_{T O P O}\right)}}
$$

i) For $E_{m}=0$, we have $K_{\text {film }} / K_{\mathrm{TOPO}}=1.75$.

ii) For $E_{m}=K_{m}$, we have $K_{\text {film }} / K_{\mathrm{TOPO}}=2.76$.

\subsection{New Christensen Model}

This model predicts ${ }^{7}$

$$
\begin{gathered}
\frac{E_{\text {film }}}{E_{\text {TOPO }}}=\frac{1-\frac{\left(1-5 v_{\text {TOPO }}\right)}{2\left(4-5 v_{\text {TOPO }}\right)} \rho}{1-2 \rho} \\
\frac{K_{\text {film }}}{K_{\text {TOPO }}}=\frac{1+\left(\frac{1-5 v_{\text {TOPO }}}{1+v_{\text {TOPO }}}\right) \rho}{1-2 \rho}
\end{gathered}
$$


i) For $v=0.1, E_{\text {film }} / E_{\mathrm{TOPO}}=6.92$ and $K_{\text {film }} / K_{\mathrm{TOPO}}=8.5$, which gives $E_{\text {film }} /\left(1-v_{\text {film }}\right)=3.96$ $E_{\mathrm{TOPO}} /\left(1-v_{\mathrm{TOPO}}\right)$.

ii) For $v=0.33, E_{\mathrm{film}} / E_{\mathrm{TOPO}}=7.6$ and $K_{\mathrm{film}} / K_{\mathrm{TOPO}}=5.6$, which gives $E_{\mathrm{film}} /\left(1-v_{\text {film }}\right)=$ $9.1 E_{\mathrm{TOPO}} /\left(1-v_{\mathrm{TOPO}}\right)$.

iii) For $v=0.5, E_{\mathrm{film}} / E_{\mathrm{TOPO}}=8.7$ and $K_{\mathrm{film}} / K_{\mathrm{TOPO}}=4.1$, which gives $E_{\mathrm{film}} /\left(1-v_{\text {film }}\right)=$ $8.7 E_{\mathrm{TOPO}} /\left(1-v_{\mathrm{TOPO}}\right)$

\subsection{Overall Conclusions}

On the basis of the first four models, we use $E_{\text {film }} /\left(1-v_{\text {film }}\right)=2.75 E_{\mathrm{TOPO}} /\left(1-v_{\mathrm{TOPO}}\right)$. The last model gives very different predictions because it assumes the capping ligands are randomly distributed — which would suggest direct contact of some cores, and this is important in this model---but the ligands are not distributed in this manner because they are bound to the cores.

\section{References}

1. Anastassakis, E.; Pinczuk, A.; Burstein, E.; Pollak, F. H.; Cardona, M., Solid State Commun. 1970, 8, 133.

2. Geurts, J., Prog. Crystal Growth and Charact. 1996, 32, 185.

3. Simmons, G.; Wang, H., Single Crystal Elastic Constants and Calculated Aggregate Properties: A Handbook. 2nd ed.; M.I.T Press: Cambridge, MA, 1971.

4. $\quad$ Halpin, J. C.; Kardos, J. L., Polym. Eng. Sci. 1976, 16, 344.

5. $\quad$ Cohen, L. J.; Ishai, O., J. Composite Mat. 1967, 1, 390.

6. Christensen, R. M., J. Mech. Phys. Solids 1990, 38, 379.

7. Christensen, R. M., Composites Part B: Eng. 2004, 35, 475. 